hep-th/9809122

EFI-98-34

TIFR/TH/98-30

\title{
String Islands
}

\author{
Atish Dabholkar \\ Department of Theoretical Physics \\ Tata Institute of Fundamental Research \\ Homi Bhabha Road, Mumbai, India 400005 \\ Jeffrey A. Harvey \\ Enrico Fermi Institute and Department of Physics \\ University of Chicago, 5640 Ellis Avenue \\ Chicago, IL 60637 U.S.A.
}

\begin{abstract}
We discuss string theories with small numbers of non-compact moduli and describe constructions of string theories whose low-energy limit is described by various pure supergravity theories.
\end{abstract}

September 1998 


\section{Introduction}

One of the main obstacles preventing more direct phenomenological applications of string theory is the problem of vacuum degeneracy. One aspect of this problem is the presence of massless scalar fields or moduli in compactifications of string theory. These moduli govern the shape and size of the compactification space as well as the value of the coupling constant in string theory and correspond to massless fields in spacetime. There are stringent constraints on the presence of such massless scalars in the real world, so it is usually assumed that masses are generated for moduli fields by whatever mechanism breaks supersymmetry in string theory. A related problem is that the moduli fields, particularly the dilaton which governs the value of the coupling constant, tend to run off to infinity in known mechanisms for supersymmetry breaking, leaving one with no vacuum at all except at zero coupling [1]. Even in a cosmological situation the presence of moduli is problematic [2]. Thus it is interesting to consider string theories with few or no moduli.

Another reason why theories with few moduli are of interest has to do with speculative proposals for a solution to the cosmological constant problem [3, 4, 5]. In [3] it was suggested that the one-loop contribution to the cosmological constant might vanish in certain special theories where the left and right-moving contributions are chiral with respect to an AtkinLehner symmetry. This naturally leads to asymmetric orbifold constructions and hence theories with a reduced number of moduli. In [4] it was proposed that the cosmological constant could vanish if our four dimensional world arises as a strong coupling limit of a three-dimensional world. In three dimensions supersymmetry can enforce a vanishing cosmological constant without imposing degeneracy between fermion and boson masses [6]. It seems quite likely that this mechanism, if it works at all, could only work in a theory which cannot be continuously connected to higher dimensional theories by varying moduli fields. This is because one could first go to a higher dimensional theory and then take the strong coupling limit which is known in many cases not to lead to supersymmetry breaking. Therefore one is again interested in theories which are free of moduli other than the dilaton or at least free of non-compact geometrical moduli which take one to higher dimensions. For apparently different reasons small numbers of moduli also entered into the proposal of [5] based on the AdS/CFT correspondence. In this case one wants the theory to contain Reissner-Nordstrom black holes with $A d S_{2}$ near horizon geometry. The $A d S_{2}$ symmetry is spoiled by the presence of moduli which couple to the gauge fields under which the black hole is charged so again one is interested in theories with few moduli. 
One final motivation for studying such theories is to improve our understanding of the moduli space of string compactifications with given spacetime supersymmetry. Of particular interest is the question of whether this moduli space is connected. In [7] it was pointed out that many Calabi-Yau vacua which were previously thought to be disconnected are in fact related via conifold transitions. More generally, if we take the size of the manifold of compactification to be very large, then locally the physics looks ten dimensional. We will give examples here of string vacua which appear to be isolated and are not continuously connected via vacuum configurations to geometrical compactifications of string theory. These theories have no geometrical moduli and moreover are self-dual so that even strong coupling does not relate them to higher-dimensional theories. We refer to these vacua as "String Islands." They are higher-dimensional supersymmetric versions of the "Ginsparg Archipelago" of $c=1$ CFT [8].

The basic idea behind our construction has been known for a long time: one can remove moduli by twisting a theory by a symmetry which exists only at special values of the moduli [9]. There is a great deal of literature on this subject; the novelty here as far as we know is that we construct theories with no moduli other than the dilaton and use string duality to argue that the strong coupling limit does not take one back to a higher-dimensional theory. Thus one can argue that these theories are truly isolated in that no variation of the moduli connects them to higher dimensional theories. Similar constructions appear in [10] in the context of two-dimensional asymmetric orbifolds where it was argued that varying certain radial moduli would lead to pure supergravity theories in higher dimensions. In such constructions one must ensure that no new moduli appear as some radii are taken to infinity. Some of the theories we construct do have a number of moduli, but many of these are compact and so do not take us back to a higher-dimensional theory.

It is also interesting to ask about theories which contain no moduli at all, not even the dilaton. Such theories exist in compactifications to two dimensions [11] and of course the most famous example of such a theory is M theory which arises as the strong coupling limit of IIA string theory. M theory is described at low-energies by eleven-dimensional supergravity. There is by now good evidence that $M$ theory exists beyond the low-energy approximation and there is a proposal for a more complete formulation of $\mathrm{M}$ theory [12] which has passed a number of non-trivial tests [13, 14]. We will comment at the end of this paper on the possibility of other theories without any moduli at all. For most of the paper we focus on perturbative string constructions in dimensions four or greater which 
therefore always contain a dilaton. The possibility of constructing moduli free theories by modding out simultaneously by $S$ and $T$ dualities and some non-perturbative aspects of theories with few moduli have been discussed in [15] .

In searching for theories with few moduli it is not completely clear what criteria to impose. The simplest and most stringent compatible with supersymmetry and the one we will impose in this paper is to try to obtain pure supergravity theories without matter fields as the low-energy limit of string constructions. Many pure supergravity theories are inconsistent because of anomalies. Of the non-anomalous pure supergravity theories we have been able to obtain all via string constructions except for one in $D=8$ and one in $D=7$.

In the next section we summarize the consistent pure supergravity theories and a few of their properties. The following section contains a set of explicit constructions of most of these theories. We end with some general comments and speculations.

\section{Consistent Pure Supergravities}

We will refer to a supergravity theory without matter multiplets as pure supergravity. Some pure supergravities of course allow the addition of matter multiplets while others (such as $D=11$ supergravity) do not, but we will not make any distinction between these two cases in what follows. Pure supergravity theories in $D$ dimensions are uniquely specified by the number of supersymmetries $N$. In six and ten dimensions we must specify the number of supersymmetries of each chirality $\left[N_{L}, N_{R}\right]$. However there does not seem to be a uniform convention for counting supersymmetries because of various reality conditions. We will therefore denote theories by $D, N$, and $N_{s}$ with $N_{s}$ the number of component real supersymmetries, so for example the low-energy limit of M-theory is denoted by $(11,1,32)$. A useful reference on the possible supergravity theories is [16].

All supergravities in $D<11$ dimensions with $N_{s}=32$ arise from toroidal compactification of $\mathrm{M}$ theory and therefore have moduli which take one back to eleven dimensions [17].

All pure supergravity theories with $N_{s}<16$ and $D \geq 4$ are either inconsistent due to anomalies $((6,[1,0], 8))$ or have no scalars at all in the supergravity multiplet $((5,1,8)$, $(4,3,12),(4,2,8),(4,1,4))$. The latter theories are interesting since they could in principle lead to lower-dimensional theories with no moduli but because they have no dilaton they cannot be obtained from perturbative string constructions. 
Of the remaining pure supergravities in $D \geq 4,(10,[1,0], 16),(6,[2,1], 24)$ and $(6,[2,0], 16)$ have perturbative gravitational anomalies and so are inconsistent. The $(9,1,16)$ theory is also inconsistent due to a global gravitational anomaly. To see this note first that a single Majorana spinor in $D=8 k+1$ dimensions has a global gravitational anomaly [18,19]. On the other hand, we know that reduction of the $D=10,(1,0)$ heterotic theory with 496 vector multiplets on $S^{1}$ gives a $D=9, N=1$ theory with 497 gauge supermultiplets, each containing a single Majorana fermion. Since this theory is consistent, it must be the case that the pure supergravity theory has a global gravitational anomaly which cancels the anomaly of the odd number of Majorana fermions in the gauge supermultiplets.

We are then left with the following list of consistent pure supergravities in $D \geq 4$ which have at least one scalar in the supergravity multiplet and cannot be obtained from toroidal compactification of M theory:

$$
\begin{aligned}
\left(D, N, N_{s}\right)= & (8,1,16),(7,1,16),(6,[1,1], 16),(5,3,24), \\
& (5,2,16),(4,6,24),(4,5,20),(4,4,16)
\end{aligned}
$$

One family of theories consists of the five theories with 16 supercharges in $D=$ $4,5,6,7,8$. The $D=5,6,7,8$ theories all have only a single real scalar field in the supergravity multiplet and thus in perturbative string theory can only arise in theories where all geometrical moduli are frozen and the dilaton is the single scalar in the supergravity multiplet. The $D=4$ theory has two scalars which comprise the dilaton/axion and thus we expect the same to be true for this theory. A reduction on $S^{1}$ of these theories in D dimensions yields the $D-1$ theory with $N_{s}=16$ with a single vector multiplet in addition to the supergravity multiplet.

A second family consists of the two theories with 24 supercharges, the $D=5, N=3$ theory and the $D=4, N=6$ theory. The $D=5, N=3$ theory contains 14 scalars, two of which are non-compact while the $D=4, N=6$ theory contains 30 scalars, three of which are non-compact. These theories are apparently related by reduction on an $S^{1}$ since the field content of the $D=4$ theory is the same as the reduction on $S^{1}$ of the $D=5, N=3$ theory. This suggests that it should be possible to obtain the $D=5, N=3$ theory as a limit of the $D=4, N=6$ theory, either by varying a geometrical modulus or by going to strong coupling.

The $D=4, N=5$ theory is the odd man out, being the only theory with 20 supercharges. The supergravity multiplet contains ten scalars, but only one of these is 
non-compact so again all moduli which would correspond to going to large radius of the internal space have been frozen.

The structure of the moduli space for these theories will be discussed in more detail in the following section in the context of explicit string constructions.

\section{Explicit Constructions}

We now turn to explicit constructions of string theories with pure supergravity theories as their low-energy limits. We will construct these theories using asymmetric orbifold constructions, although there are undoubtedly other constructions of some of these theories involving free fermions, tensor products of minimal models, or orientifolds.

First we recall a few basic facts about asymmetric orbifold constructions [20]. In this paper we deal primarily with orbifolds of Type-II string theory. We thus start with a toroidal compactification of Type-II string on a d-dimensional torus. At special points in the Narain moduli space of such compactifications we can obtain theories with purely left or right moving symmetries. These occur at points in the Narain moduli space where some of the T-duality symmetries have fixed points. One very useful construction of such special points proceeds as follows [20]. We choose a simply laced Lie algebra $\mathcal{G}$ of rank $d$ and define the lattice $\Gamma^{d, d}(\mathcal{G})$ [21] as

$$
\Gamma^{d, d}(\mathcal{G})=\left\{\left(p_{L}, p_{R}\right)\right\}, \quad p_{L}, p_{R} \in \Lambda_{W}(\mathcal{G}), \quad p_{L}-p_{R} \in \Lambda_{R}(\mathcal{G})
$$

with $\Lambda_{R}$ and $\Lambda_{W}$ the root and weight lattices of $\mathcal{G}$ respectively. The resulting theory has purely left (and right) moving symmetries given by elements of the Weyl group $\mathcal{W}(\mathcal{G})$, and in order to act correctly on fermion fields these must also be elements of $\operatorname{Spin}(d)$. The general transformation is thus of the form

$$
\left|p_{L}, p_{R}\right\rangle \rightarrow e^{2 \pi i\left(p_{L} \cdot v_{L}-p_{R} \cdot v_{R}\right)}\left|g_{L} p_{L}, g_{R} p_{R}\right\rangle
$$

where $v=\left(v_{L}, v_{R}\right)$ is a shift vector and $g_{L}$ and $g_{R}$ lie in the intersection of $\mathcal{W}(\mathcal{G})$ with $\operatorname{Spin}(d)$. The right and left-moving fermions must also be twisted by $g_{R, L}$ in order to preserve the world-sheet supersymmetry.

Classifying such orbifolds amounts to classifying conjugacy classes of the Weyl groups of rank $d$ simply laced Lie algebras and then for elements of each conjugacy class determining the allowed shift vectors which are consistent with modular invariance. In what follows 
we will use the classification of and notation for Weyl group conjugacy classes developed by Carter [22]. Useful tables of these conjugacy classes can be found in [23,24]. We will often try to choose shift vectors which give positive vacuum energy in the twisted sectors in order to ensure that there are no massless states coming from the twisted sectors. In doing this it is important to remember that in twisted sectors the momenta live in the lattice $I^{*}$ which is dual to the lattice left invariant by the twist. Thus we will want to choose shift vectors which are not in $I^{*}$. A necessary condition for modular invariance of abelian orbifolds is level-matching [25]. For $\mathbf{Z}_{n}$ orbifolds, level-matching is ensured if in every sector there are states for which $n\left(E_{R}-E_{L}\right)=0 \bmod 1$ where $E_{L, R}$ are the left and right-moving energies. Equivalently, there must be physical states in every twisted sector for every ground state of definite momentum and winding. This condition is known to be sufficient for modular invariance at one loop [25]. In fact it suffices to check level matching for the ground state rather than for all momentum and winding states and to check a single mod 2 condition for elements of even order $2 n$ :

$$
p g^{n} p=0 \quad \bmod 2
$$

for all $p \in \Gamma^{d, d}$. In what follows we will mostly use odd order twists for which it suffices to check level matching for the ground state.

Conditions for modular invariance at higher loops have been analyzed in [26] where it is shown that one-loop level matching is not sufficient to guarantee level matching for theories with a non-Abelian point group. Our theories all have Abelian point groups and so we do not expect any problems with higher loop modular invariance, but it is not clear to us that the analysis of [26] guarantees higher loop modular invariance for abelian asymmetric orbifolds which do not have a simple fermionic description.

As discussed above we will focus on consistent minimal supergravities with $16 \leq N_{s}<$ 32. Theories with $N_{s}=16$ occur in dimensions $D=4,5,6,7,8$. These theories contain $8(D-2)$ physical boson and fermion degrees of freedom and have half of the maximal supersymmetry. In Type-II string, 16 supercharges come from the left-movers and 16 come from the right-movers. This suggests that one can construct an asymmetric orbifold with a left-moving twist which lies in $O(10-D)$ but not in a subgroup and which thus breaks half of the spacetime supersymmetry coming from the left-movers and removes all Narain moduli from the untwisted sector. If we accompany the twist by a right-moving shift which prevents the occurrence of massless states in the twisted sector we clearly get the minimal supergravity spectrum for theories with $N_{s}=16$. 
If the left-moving twist lies in $S U(3)$ or in $S U(2)$, then four or eight left-moving supersymmetries are preserved respectively, giving us 20 or 24 supersymmetries in all. In some examples that we discuss below, additional supersymmetries come from the twisted sector if the the twists are not accompanied by any shifts.

As we move down in dimension there are both more moduli that must be projected out by the orbifold and larger orbifold groups and more choices of shift vector that can be used in the construction. It is not obvious a priori, but it will turn out that going down in dimension in fact makes it easier to remove the moduli and that as we move up in dimension it becomes more difficult until we reach $D=7$ and $D=8$ where it seems unlikely that asymmetric orbifold constructions of the pure supergravity theories exist. This will become clearer as we proceed.

We now consider such orbifolds on a case by case basis. In what follows we write the roots of $A_{n}$ in an $n+1$ dimensional orthonormal basis $\left\{e_{i}, i=1 \ldots n+1\right\}$ as $\left\{\left(e_{i}-e_{j}\right)\right\}$. For $D_{n}$ we write the roots in terms of $\left\{e_{i}, i=1 \ldots n\right\}$ as $\left\{ \pm e_{i} \pm e_{j}\right\}$.

\section{1. $D=4$}

\subsection{1. $N=4$}

In order to implement the orbifold discussed above we need a rank six simply laced Lie algebra and an element of the Weyl group of this Lie algebra which lies in $S O(6)$ but not in a subgroup. An inspection of the list of conjugacy classes of Weyl groups of simply laced Lie algebras [23,24] leads to many possibilities. Let $\omega$ denote a primitive nth root of unity when the Weyl group element has order $n$. We find for example that $E_{6}$ has a conjugacy class $E_{6}\left(a_{1}\right)$ of elements of order 9 with eigenvalues $\omega, \omega^{2}, \omega^{4} ; D_{6}$ has a conjugacy class $D_{6}\left(a_{2}\right)$ of elements of order 6 with eigenvalues $\omega, \omega^{3}, \omega^{5} ; A_{4} \times A_{2}$ has a conjugacy class $A_{4} \times A_{2}$ of elements of order 15 with eigenvalues $\omega^{3}, \omega^{5}, \omega^{6}$ along with many other possibilities.

a. We can construct a $D=4, N=4$ model based on the conjugacy class $E_{6}\left(a_{1}\right)$ of the $E_{6}$ Weyl group [27]. We start with the lattice $\Gamma^{6,6}\left(E_{6}\right)$. We then twist by a $Z_{9}$ element with $g_{R}=1$ and $g_{L}=\left(\omega, \omega^{2}, \omega^{4}\right)$. We accompany this by a shift with $v_{L}=0$ and

$$
v_{R}=\frac{1}{9}(1,1,-2 ; 1,1,-2 ;-1,-1,+2)
$$


Here we have written the shift vector in terms of the embedding of $A_{2}^{3} \subset E_{6}$. The action of the twist $g_{L}$ can be represented by a $3 \times 3$ matrix action on the $A_{2} \times A_{2} \times A_{2}$ planes as

$$
\left(\begin{array}{ccc}
0 & 1 & 0 \\
0 & 0 & 1 \\
\alpha & 0 & 0
\end{array}\right),
$$

where $\alpha$ is a rotation in a single $A_{2}$ plane by $2 \pi / 3$.

In this model $I=\left(0, \Lambda_{R}\left(E_{6}\right)\right), I^{*}=\left(0, \Lambda_{W}\left(E_{6}\right)\right)$ and we can check that $v_{R}$ is not in $I^{*}$, and moreover $3 v_{R}$ is also not in $I^{*}$ because $3 v_{R}$ is in the $(\mathbf{3}, \mathbf{3}, \overline{\mathbf{3}})$ conjugacy class of $A_{2}^{3}$, whereas $\Lambda_{W}\left(E_{6}\right)$ has only the $(\mathbf{3}, \mathbf{3}, \mathbf{3})$ and $(\overline{\mathbf{3}}, \overline{\mathbf{3}}, \overline{\mathbf{3}})$ conjugacy classes.

b. It is also possible to construct a heterotic string theory with pure $D=4, N=4$ supergravity as its low-energy limit using a free fermion construction as discussed in [28].

In both of these examples one obtains the $N=4$ pure supergravity multiplet that contains the graviton, four gravitini, six graviphotons, and two scalars. The only noncompact scalar is the dilaton $\phi$ which is accompanied by the axion $b$ that comes from the dualized antisymmetric tensor $B_{\mu \nu}$. Together, the scalars parameterize the coset $S L(2, \mathbf{R}) / S O(2)$. The duality group is expected to be a subgroup of $S L(2, \mathbf{Z})$. Precise determination of this duality group in each example is an important and interesting problem.

\subsection{2. $N=5$}

a. We start with a toroidal compactification of type II string theory described by the lattice $\Gamma^{6,6}\left(A_{6}\right)$ and twist by the $\mathbf{Z}_{7}$ symmetry generated by

$$
\begin{aligned}
& g_{L}=\left(\omega, \omega^{2}, \omega^{4}\right), \quad v_{L}=0 \\
& g_{R}=1, \quad v_{R}=\frac{1}{7}(1,2,-3,0,0,0,0) .
\end{aligned}
$$

In terms of orthonormal basis vectors $e_{i}, i=1, \ldots, 7 \mathrm{in} \mathbf{R}^{7}$ the weight lattice of $A_{6}$ is in the hyperplane in $\mathbf{R}^{7}$ that is orthogonal to $\sum_{i} e_{i}$. The Weyl group of $A_{6}$ is the permutation group $S_{7}$ which permutes the seven basis vectors. A $\mathbf{Z}_{7}$ subgroup of the Weyl group is generated by $e_{i} \rightarrow e_{i+1}$ for all $i$. There are seven eigenvalues given by the different seventh roots of unity. The eigenvector corresponding to eigenvalue 1 is obviously $\sum_{i} e_{i}$ which is orthogonal to the weight lattice. Therefore, we can choose a complex basis in which the remaining six eigenvalues are $\omega, \omega^{2}, \omega^{4}$ and their complex conjugates. It is also clear that $7 v_{R}$ is in the root lattice of $A_{6}$ and that $v_{R}$ is not in $I^{*}=\Lambda_{W}\left(A_{6}\right)$. 
b. Another possibility is to consider the lattice $\Gamma^{6,6}\left(A_{2}^{3}\right)$ and twist by an asymmetric $\mathbf{Z}_{3}$ symmetry generated by

$$
\begin{aligned}
& g_{L}=(\omega, \omega, \omega), \quad v_{L}=0 \\
& g_{R}=1, \quad v_{R}=\frac{1}{3}(1,-1,0 ; 1,-1,0 ; 2,-2,0) .
\end{aligned}
$$

In both these models there is no twist on the right, so all four supersymmetries from the right are preserved. On the left, the twist is in $S U(3)$, so one supersymmetry from the left is preserved and together one obtains $N=5$ supersymmetry.

In the NS-NS sector we obtain the metric $g_{\mu \nu}$, an antisymmetric tensor $B_{\mu \nu}$ which can be dualized to a scalar $a$, the dilaton $\phi$, and six vector fields. In the R-R sector we find eight additional scalars and four more vector fields. Thus the moduli consist of one non-compact scalar, the dilaton, and nine compact scalars.

The supergravity action is known to have $S U(1,5)$ symmetry. The ten vector fields transform in the 10 (self-dual antisymmetric rank-three tensor) of $S U(1,5)$. The classical moduli space parametrized by the ten scalars is locally the coset $S U(1,5) / U(5)$.

Since there are no states from the twisted sectors, one can determine the symmetry group purely from group theory. Our starting point is Type-II theory compactified on a 6-torus. The moduli space is $E_{7}(\mathbf{Z}) \backslash E_{7}(\mathbf{R}) / S U(8)$ where the $E_{7}$ is in the maximally split form. The duality group $E_{7}(\mathbf{Z})$ maps a generic point $x$ of the coset $E_{7}(\mathbf{R}) / S U(8)$ to some other point $x^{\prime}$ of the coset. If a subgroup $G_{x}$ of $E_{7}(\mathbf{Z})$ leaves $x$ invariant then it would be a symmetry of the theory at $x$. One can then orbifold the theory at $x$ with the orbifold group $G_{x}$ to obtain a new theory. $G_{x}$ is obviously a discrete subgroup of the isotropy group $S U(8)$. In our case because the left-moving twist preserves four supersymmetries, it is a subgroup also of $S U(3)$. In the absence of twisted states, we expect that the symmetry group of the orbifold theory will be the subgroup of $E_{7}(\mathbf{R})$ that commutes with $S U(3)$. It is somewhat subtle to see that one obtains the correct real form $S U(1,5)$ and not, say, $S U(2,4)$. One useful observation is that, in $S U(8)$, which is the maximal compact subgroup of $E_{7}$, the centralizer of $S U(3)$ is $U(5)$. The symmetry group therefore must contain $U(5)$ as a compact subgroup. If we decomose the adjoint representation of $E_{7}$ in terms of $S U(8) \supset S U(3) \times U(5)$ representations, and keep only those states that are invariant under the $S U(3)$, then the remaining $U(5)$ representations properly combine into $S U(1,5)$ adjoint representation. Using these facts one can easily determine, in agreement with the supergravity considerations, that the symmetry group is indeed $S U(1,5)$. 
One can ask what the quantum moduli space is. Supersymmetry prevents any quantum corrections, so the question is really only about global identifications or the U-duality group. At the level of group theory it seems natural to conjecture that the U-duality group is $S U(1,5, \mathbf{Z})$. Physically this may not be true because, in general, duality does not commute with orbifolding. One supporting piece of evidence is that the quantized electric and magnetic charges certainly exist in the theory. They transform as $\mathbf{2 0}$ of $S U(1,5)$ and satisfy the Dirac quantization condition. One way to define the integral form $S U(1,5, \mathbf{Z})$ is to note that the $\mathbf{2 0}$ representation of $S U(1,5)$ is symplectic. The group $S p(20, \mathbf{Z})$ has a natural action on the lattice of electric and magnetic charges. One might therefore define $S U(1,5, \mathbf{Z})$ as the intersection of $S p(20, \mathbf{Z})$ and $S U(1,5)$. This conjecture can be tested by analyzing the spectrum of dyonic bound states in the theory.

\subsection{3. $\mathrm{N}=6$}

We know two ways to obtain this theory.

a. The first is closely related to a model discussed in 29] and is obtained by a $\mathbf{Z}_{2}$ asymmetric twist on $\Gamma^{4,4}\left(D_{4}\right)$ 1 . The $\mathbf{Z}_{2}$ acts as -1 on the four left-moving coordinates of $\Gamma^{4,4}$, as a shift by half a lattice vector on the right, and this action is accompanied by an asymmetric shift along one component of a $\Gamma^{2,2}$. This theory has a limit where the radius of the untwisted and unshifted direction goes to infinity and in this limit it gives the $D=5, N=3$ theory. The local moduli space of the $D=5, N=3$ theory is

$$
\frac{S U^{*}(6)}{U S p(6)}
$$

The theory thus has two non-compact moduli which in this construction correspond to the dilaton and a modulus which is the radius of the shifted $S^{1}$. Since states odd under the twist and with odd momenta on this $S^{1}$ are physical, by going to infinite radius we recover the $D=6, N=(2,2)$ theory and we can perturb all the way back to $D=10$.

b. The second construction is the theory with an asymmetric $\mathbf{Z}_{3}$ twist. We take a lattice $\Gamma^{6,6}\left(A_{2}^{3}\right)$ and twist by

$$
\begin{array}{lrl}
g_{L} & =(\omega, \omega, \omega), & v_{L}=0 \\
g_{R}=1, & v_{R}=0 .
\end{array}
$$

1 The model discussed in [29] had the same orbifold action but started with the lattice $\Gamma^{4,4}\left(A_{1}^{4}\right)$. As pointed out to us by E. Silverstein, this model is not modular invariant because it does not satisfy the mod 2 condition (3.3). 
This is rather similar to (3.7) that gave us $N=5$ theory except that there is no shift; as a result now there are additional massless states in the twisted sector. We get $N=5$ supergravity from the untwisted sector as in (3.7). To find the number of twisted sectors, note that $g_{L}$ leaves $\left(0, p_{R}\right)$ invariant. So, the invariant lattice $I$ is the root lattice of $S U(3)^{3}$, and $I^{*}$ is therefore the weight lattice of $S U(3)^{3}$. The number of twisted sectors is $D \equiv$ $\sqrt{\operatorname{det}\left(1-\theta_{L}\right) /\left|I^{*} / I\right|}=\sqrt{27 / 27}=1$. The single twisted sector contributes an additional gravitino multiplet. Together, we obtain the gravity multiplet of $N=6$ supersymmetry.

In this construction there is no obvious radial modulus to vary so it is not clear if this theory has a limit which gives the $D=5, N=3$ theory and if so whether the limit is perturbative or involves strong coupling.

The bosonic spectrum of $N=6$ supergravity contains, in addition to the graviton, 32 vector fields and 30 scalars. The symmetry group in this case is $S O^{*}(12)$. The vectors transform in the 32-dimensional spinor representation. The scalars parameterize the coset $S O^{*}(12) / U(6)\left[30\right.$. Because the real rank of $S O^{*}(12)$ is three, there are three non-compact moduli.

\section{2. $D=5$}

\subsection{1. $N=2$}

We start with type II string theory at the point in Narain moduli space defined by the lattice $\Gamma^{5,5}\left(D_{5}\right)$. The Weyl group has a conjugacy class $D_{5}\left(a_{1}\right)$ of elements of order 12 with a pair of complex eigenvalues $\omega^{2}, \omega^{3}$ and a single real eigenvalue of $-1=\omega^{6}$. The shift vector $v_{R}=(1,1,2,3,3) / 12$ satisfies level matching and neither $v_{R}$ nor $n v_{R}, n<12$ lie in $I^{*}$. This asymmetric orbifold thus leads to pure $N=2$ supergravity in $D=5$. The only scalar in the spectrum is the dilaton which parameterizes the positive real line $\mathbf{R}^{+}$.

\subsection{2. $N=3$}

This theory was discussed earlier as a perturbative large radius limit of a $D=4, N=6$ theory. 


\section{3. $D=6$}

To obtain $N=(1,1)$ pure supergravity, we start with type II string theory at the point in Narain moduli space defined by the lattice $\Gamma^{4,4}\left(A_{4}\right)$. The Coxeter element of $A_{4}$ is order 5 and has eigenvalues $\omega, \omega^{3}$. This is in $S O(4)$ but not in $S U(2)$ so twisting by this element on the left breaks all the left-moving supersymmetries. The shift vector $v_{R}=(0,1,-1,2,-2) / 5$ satisfies level matching and is not in $I^{*}$ so there are no massless states in the twisted sectors. The spectrum of this orbifold is that of pure $N=(1,1)$ supergravity. Again, the dilaton parameterizes the positive real line $\mathbf{R}^{+}$.

\section{4. $D=7$ and 8}

These construction seems to fail when we get to seven or eight dimensions because we cannot find appropriate shift vectors. For $D=8$ we can give an exhaustive demonstration that there are no asymmetric orbifold constructions of type II string theory leading to the $(8,1,16)$ theory in the low-energy limit.

We can classify the possible asymmetric orbifolds as follows. We start with a compactification on $T^{2}$ with moduli space $O(2,2, \mathbf{Z}) \backslash O(2,2) / O(2) \times O(2)$, or equivalently

$$
[S L(2, \mathbf{Z}) \backslash S L(2, \mathbf{R}) / U(1) \times S L(2, \mathbf{Z}) \backslash S L(2, \mathbf{R}) / U(1)] / \mathbf{Z}_{2} .
$$

We can twist by elements of the T-duality group if we are at a point in the moduli space where these elements preserve the lattice and therefore have fixed points acting on the above coset. Let $\sigma$ and $\tau$ be the modular coordinates on each component of (3.10). Then following [31] we can write the $\Gamma^{2,2}$ lattice using a complex basis as

$$
\Gamma^{2,2}=\frac{1}{\sqrt{2 \Im \sigma \Im \tau}} \mathbf{Z}\left(\begin{array}{l}
1 \\
1
\end{array}\right) \oplus \mathbf{Z}\left(\begin{array}{c}
\bar{\sigma} \\
\sigma
\end{array}\right) \oplus \mathbf{Z}\left(\begin{array}{c}
\tau \\
\tau
\end{array}\right) \oplus \mathbf{Z}\left(\begin{array}{c}
\bar{\sigma} \tau \\
\sigma \tau
\end{array}\right)
$$

Fixed points occur at the orbifold point $\sigma=i, \tau=i$ which has an enhanced $\left(Z_{4} \times Z_{4}\right) \ltimes Z_{2}$ symmetry, at $\sigma=\rho, \tau=\rho$ with $\rho=e^{2 \pi i / 3}$ which has an enhanced $Z_{3}$ symmetry and at $\sigma=i, \tau=\rho$ (or vice versa) which has a $Z_{12}$ symmetry which acts quasicrystallographically [11] as

$$
\left(\begin{array}{l}
z_{1} \\
z_{2}
\end{array}\right) \rightarrow\left(\begin{array}{cc}
-i \rho & 0 \\
0 & i \rho
\end{array}\right)\left(\begin{array}{l}
z_{1} \\
z_{2}
\end{array}\right)
$$

The $Z_{12}$ twist breaks all the supersymmetry and so does not yield the theory in question. Similarly, twists by a subgroup of $Z_{12}$ at the point $\sigma=i, \tau=\rho$ also break all the supersymmetry since they act both on the left and right. At the point $\tau=\sigma=i$ the 
$Z_{4}$ or $Z_{2}$ abelian subgroups of $\left(Z_{4} \times Z_{4}\right) \ltimes Z_{2}$ do not satisfy the mod two condition (3.3). At the $Z_{3}$ symmetric point one can easily classify the possible shift vectors of order three and find that shift vectors compatible with modular invariance always lie in $I^{*}$ and thus lead to additional massless fields in the twisted sector. We have not tried to perform an exhaustive search of orientifold constructions or heterotic constructions of this theory, but they seem unlikely to exist. Note that no such constructions were found in the detailed search of heterotic free fermion constructions carried out in [28].

We have not tried to carry out a similar classification of possible $D=7$ constructions, but the obvious possibilities all fail to construct the $(7,1,16)$ theory.

It might be possible to obtain these theories in the context of F-theory [32]. In [33] an extension of F-theory is considered where the monodromy of the coupling constant field $\tau$ is in a subgroup of $S L(2, \mathbf{Z})$ because of a nontrivial background of the 2 -form $B_{\mu \nu}$ field. Such compactifications also lead to vacua where some of the moduli are frozen. More generally, it would be interesting to know whether some or all of the string islands considered in this paper can also be obtained as F-theory compactifications.

\section{General Comments and Speculations}

We have constructed all but two of the consistent pure supergravity theories in $D \geq$ 4 as the low-energy limit of asymmetric orbifold string constructions. Several of these theories have no moduli other than the dilaton and we expect that they are self-dual so that the strong coupling limit simply leads again to the same theory. For the theories with $N_{s}=16$ in $D=4,5,6$ there is no consistent higher dimensional theory with the right spectrum that one could obtain by taking the strong coupling limit. The strong coupling limit must therefore have the same low-energy spectrum, although possibly with a different construction leading to a different massive spectrum. For other cases such as the $(4,6,24)$ theory constructed in 3.1.3.b the strong coupling limit is not clear. In all these cases it would be interesting to study the non-perturbative D-brane spectrum in order to determine the full U-duality group and hence the strong coupling limit.

Finally, part of the motivation for this work was the possibility of constructing lowerdimensional versions of $\mathrm{M}$ theory, that is supersymmetric theories with no moduli at all which at low-energies are described by one of the moduli-free pure supergravities $(5,1,8)$,

$(4,3,12),(4,2,8)$ or $(4,1,4)$. The $(5,1,8)$ theory in particular has many similarities to $\mathrm{M}$ theory [34,35, 36]. It is tempting to speculate that such theories might be obtained as 
the strong coupling limit of theories in $D=4$ or $D=3$ which have only the dilaton as a modulus. In particular, the $(5,1,8)$ theory could arise as the strong coupling limit of a $D=4, N=2$ theory with a single vector-multiplet. In fact, given such a theory, this would seem to be the simplest candidate for the strong coupling limit. Needless to say, we have not yet been able to construct such an asymmetric orbifold although there does not seem to be any fundamental reason why such a construction should not exist [37]. Of course, given such a construction one would still need to exhibit evidence for a KaluzaKlein spectrum of soliton bound states. For the time being this theory remains a "Fantasy Island."

\section{Acknowledgements}

It is a pleasure to thank L. Dixon for helpful discussions and for sharing some of his constructions, and S. Ferrara, A. Klemm, G. Moore, M. S. Raghunathan and E. Silverstein for valuable discussions. We would like to acknowledge the hospitality of the Institute for Theoretical Physics at Santa Barbara, and thank the organizers of the workshop on 'Duality in String Theory' for inviting us. A. D. would like to acknowledge the hospitality of the Enrico Fermi Institute at the University of Chicago where some of this work was completed. This work was supported in part by the National Science Foundation under Grant No. PHY96-00697 and Grant No. PHY94-07194. 


\section{References}

[1] M. Dine and N. Seiberg, "Couplings and Scales in Superstring Models," Phys. Rev. Lett. 55 (1985) 366.

[2] For a review see T. Banks, "SUSY Breaking, Cosmology, Vacuum Selection and the Cosmological Constant in String Theory," hep-th/9601151.

[3] G. Moore, "Atkin-Lehner Symmetry," Nucl. Phys. B293 (1987) 139., Erratum ibid. B299 (1988) 847.

[4] E. Witten, "Strong Coupling and the Cosmological constant," Mod. Phys. Lett. A10 (2153) 1995, hep-th/9506101.

[5] S. Kachru, J. Kumar and E. Silverstein, "Vacumm Energy Cancellation in a Nonsupersymmetric String," hep-th/9807076.

[6] E. Witten, "Is Supersymmetry Really Broken," Int. J. Mod. Phys.A10 (1995) 1247, hep-th/9409111.

[7] A. Strominger, B. Greene and D. Morrison, "Black Hole Condensation and the Unification of String Vacua," Nucl. Phys. B451 (1995) 109, hep-th/9504145.

[8] P. Ginsparg, "Curiosities at $c=1$," Nucl. Phys. B295 (1988) 153.

[9] M. Mueller and E. Witten, "Twisting Toroidally Compactified Heterotic Strings with Enlarged Symmetry Groups," Phys. Lett. 182B (1986) 28.

[10] S. Chaudhuri and D. A. Lowe, "Monstrous String-String Duality," Nucl. Phys. B469 (1996) 21 , hep-th/9512226.

[11] J. A. Harvey. G. Moore and C. Vafa, "Quasicrystalline Compactification," Nucl. Phys. B304 (1988) 269.

[12] T. Banks, W. Fischler, S. Shenker and L. Susskind. "M Theory as a Matrix Model: A Conjecture," Phys. Rev. D55 (1997), hep-th/9610043.

[13] T. Banks, "Matrix Theory," Nucl. Phys. Proc. Suppl. 67 (1998) 180, hep-th/9710231.

[14] D. Bigatti and L. Susskind, "Review of Matrix Theory," hep-th/9712072.

[15] M. Dine and E. Silverstein, "New M-theory Backgrounds with Frozen Moduli," hepth/9712166.

[16] Supergravities in Diverse Dimensions, Eds. A. Salam and E. Sezgin, North Holland and World Scientific, 1989.

[17] E. Witten, "String Theory Dynamics in Various Dimensions," Nucl. Phys. B443 (1995) 85, hep-th/9503124.

[18] L. Alvarez-Gaumé and E. Witten, "Gravitational Anomalies," Nucl. Phys. B234 (1984) 269.

[19] E. Witten, "Global Gravitational Anomalies," Commun. Math. Phys. Commun. Math. Phys. 100 (1985) 197.

[20] K. Narain, M. Sarmadi, and C. Vafa, "Asymmetric Orbifolds," Nucl. Phys. B288 (1987) 551. 
[21] K. S. Narain, "New Heterotic String Theories in Uncompactified Dimension < 10," Phys. Lett. 169B (1986) 41.

[22] R. W. Carter, "Conjugacy Classes in the Weyl Group," Comp. Math. 25 (1972) 1.

[23] P. Bouwknegt, "Lie Algebra Automorphisms, the Weyl Group, and Tables of Shift Vectors," J. Math. Phys. 30(3) (1989) 571.

[24] W. Lerche, A. N. Schellekens and N. P. Warner, "Lattices and Strings," Phys. Rept. 177 (1989) 1.

[25] C. Vafa, "Modular Invariance and Discrete Torsion on Orbifolds," Nucl. Phys. B273 (1986) 592.

[26] D. Freed and C. Vafa, "Global Anomalies on Orbifolds," Comm. Math. Phys. 110 (1987) 349.

[27] This example is due to L. Dixon, unpublished.

[28] S. Chaudhuri, G. Hockney, and J. Lykken, "Maximally Supersymmetric String Theories in $D<10$," Phys. Rev. Lett. 75 (1995) 2264, hep-th/9505054.

[29] A. Sen and C. Vafa, "Dual Pairs of Type-II String Compactification, Nucl. Phys. B455 (1995) 165, hep-th/9508064.

[30] L. Adrianopoli, R. D' Auria, and and S. Ferrara, "U-duality and Central Charges in Various Dimensions Revisited", Int. J. Mod. Phys. A13 (1998) 431, hep-th/9612105.

[31] R. Dijkgraaf, E. Verlinde and H. Verlinde, " $c=1$ Conformal Field Theory on Riemann Surfaces," Comm. Math. Phys. 115 (1988) 649.

[32] C. Vafa, "Evidence for F-theory," Nucl. Phys. B469 (1996) 403, hep-th/9602022.

[33] P. Berglund, A. Klemm, P. Mayr, and S. Theisen, "On Type IIB Vacua With Varying Coupling Constant," hep-th/9805189.

[34] E. Cremmer in "Cambridge 1980, Proceedings, Superspace and Supergravity," eds. S. W. Hawking and M. Rocek, Cambridge University Press, 1981.

[35] A. H. Chamseddine and H. Nicolai, Phys. Lett. 96B (1980) 89.

[36] S. Mizoguchi and N. Ohta, "More on the Similarity between $D=5$ Simple Supergravity and M Theory," hep-th/9807111.

[37] A. Dabholkar and J. Harvey, in progress, 